\title{
Optimal Method of Runge-Kutta of Order 5
}

\author{
Akpini K. A. Michaël ${ }^{1}$, Assui K Richard ${ }^{2}$, Yoro Gozo ${ }^{3} \&$ Bailly Balé $^{4}$
}

${ }^{1}$ UFR SFA Université Nangui Abrogoua d'Abobo-Adjamé, Abidjan, Côte d'Ivoire

${ }^{2}$ Département de Mathématiques et Informatique, Institut National Polytechnique Houphouët Boigny, Yamoussoukro, Côte d'Ivoire

${ }^{3}$ UFR SFA,Université Nangui Abrogoua d'Abobo-Adjamé, Abidjan, Côte d'Ivoire

${ }^{4}$ UFR Mathématiques et Informatique, Université Felix Houphouët Boigny de Cocody,Abidjan, Côte d'Ivoire

Correspondence: Akpini Kouadio Amos Michaël, UFR SFA Université Nangui Abrogoua d'Abobo-Adjamé, Abidjan, Côte d'Ivoire.

Received: December 13, 2018 Accepted: January 21, 2019 Online Published: January 24, 2019

doi:10.5539/jmr.v11n1p93 URL: https://doi.org/10.5539/jmr.v11n1p93

\begin{abstract}
The Runge-Kutta method of order 5 with 6 stages requires finding a matrix A, whose coefficients must satisfy a system of nonlinear polynomial equations. Butcher found a 5-parameter family of solutions, which displays different characteristics depending on whether $b_{2}=0$ or $b_{2} \neq 0$. This paper presents an optimal method in the case $b_{2}=0$, which is significantly better than several popular methods of order 4 .
\end{abstract}

Keywords: Runge-Kutta method, optimal, stability, estimation, error

\section{Introduction}

The methods of Runge-Kutta (RK) are one of the principal methods of numerical resolution of the issue of Cauchy for ordinary differential equations (ODE) (Hairer,Nersett \& Wanner,1990; Cassity,1969). These methods are part of the family of non separate methods. The method RK used for the resolution of ODE is characterised by two main parameters $(p ; s)$ for which $p$ indicates the order of the method and $s$ the number of steps or stages.

For the resolution of the method with $s$ stages, we must find a matrix $A$, the coefficients $b_{i}$ and $c_{i}$ for $i=1, \cdots, s$ which corresponds to a system of non linear polynomial equations. These methods are generally represented in the form of tabulation named the Butcher table (butcher,1969):

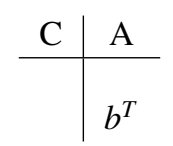

in which $A$ represents the triangular square matrix which is strictly inferior of coefficient $a_{i j}, i, j=1, \cdots, s$ for dimension $s \times s, b$ and $c$ are respectively vectors of dimension $s$ such as $b^{T}=\left(b_{1}, \cdots, b_{s}\right)$ and $c=\left(c_{1}, \cdots, c_{s}\right)^{T}$. The vector $c$ contains variables called free variables .

If the matrix $A=a_{i j}$ is such $\mathrm{t} a_{i j}=0$ for $i<j$, the method is said to be explicit(ERK) in the one hand. it is settled implicit on the over hand.

Practically, we always have

$$
c_{i}=\sum_{j=1}^{i-1} a_{i j}, \quad \sum_{i=1}^{s} b_{i}=1, \quad c_{s}=1 .
$$

For instance, the methods of Runge-Kutta of order 4 with 4 stages known under the famous name of "classical RK methods" are characterised by a square matrix $A$ for which the coefficients satisfy a system of eight (8) nonlinear polynomial equations with ten (10) variables. There are family of rational and bidimensional solutions. In these methods, the free variable usually used are $c_{2}$ and $c_{3}$ (Hairer, Nersett $\&$ Wanner,1990).

For the resolution of the Runge-Kutta method of order 5 with 6 stages, the coefficients must correspond moreover to 9 equations that is to say we obtain a system of 17 equations with 21 variables. It is known in (Hairer,Nersett \& Wanner,1990;Cassity,1969) that this system does not have any solutions. But the value $S^{2}$ designates the sum of the square values of the supplementary equations may be used to compare the efficiency of the different methods of Runge-Kutta of order 4: the best equation is the one which is near 0 .

by the way of comparison,the values of $S$ for certain methods are: 


\begin{tabular}{c|c}
\hline Method & $\mathrm{S} \approx$ \\
\hline the rule " $3 / 8 "$ & 0.015 \\
$c_{2}=c_{3}=1 / 2$ & 0.022 \\
$c_{2}=0.312 ; c_{3}=0.642$ & 0.014 \\
\hline
\end{tabular}

The value of the last line is the smallest as possible. We can see that the "error" $S$ is not too different for various methods. In the most detailled way, this issue has been studied by diverse authors, for more detail, see. (Hairer, Nersett, Wanner,1990), ch.11,3. Practically these methods of resolution do not make a success because of a slight increasing of precision and the rightness. With the methods of order 5 , the situation is different. The value of the error for these methods is indicated in the literature and the method used are practically distinguished to hundred times.

\section{The Error Function}

The method of Runge-Kutta of order 5 with 6 stages is given by the following Butcher table:

$$
\begin{array}{c|cccccc}
c_{2} & a_{21} & & & & & \\
c_{3} & a_{31} & a_{32} & & & & \\
c_{4} & a_{41} & a_{42} & a_{43} & & & \\
c_{5} & a_{51} & a_{52} & a_{53} & a_{54} & & \\
c_{6} & a_{61} & a_{62} & a_{63} & a_{64} & a_{65} & \\
\hline & b_{1} & b_{2} & b_{3} & b_{4} & b_{5} & b_{6}
\end{array}
$$

If the table (1) below definies the methods of RK of order 5 then these coefficients must correspond to a sytem of 17 equations (Hairer,Nersett, Wanner 1990;Cassity,1969). This system is presented as follows:

$\begin{array}{llll}(1) & \{b\} & = & 1 \\ (2) & \{b * \tilde{A} e\} & = & 1 / 2 \\ (3) & \left\{b * \tilde{A}^{2} e\right\} & = & 1 / 6 \\ (4) \quad\{b * \tilde{A} * \tilde{A} e\} & = & 1 / 3 \\ (5) \quad\left\{b * \tilde{A}^{3} e\right\} & = & 1 / 24 \\ (6) \quad\left\{b * \tilde{A}^{2} e * \tilde{A} e\right\} & = & 1 / 8 \\ (7) \quad\{b * \tilde{A} e * \tilde{A} e * \tilde{A} e\} & = & 1 / 4 \\ (8) \quad\{b * \tilde{A}(\tilde{A} e * \tilde{A} e)\} & = & 1 / 12 \\ (9) \quad\left\{b * \tilde{A}^{4} e\right\} & = & 1 / 120 \\ (10) \quad\left\{b * \tilde{A}\left(\tilde{A}^{2} e * \tilde{A} e\right)\right\} & = & 1 / 40 \\ (11) \quad\{b * \tilde{A}(\tilde{A} e * \tilde{A} e * \tilde{A} e)\} & = & 1 / 20 \\ (12) \quad\left\{b * \tilde{A}^{2}(\tilde{A} e * \tilde{A} e)\right\} & = & 1 / 60 \\ (13) \quad\left\{b * \tilde{A}^{3} e * \tilde{A} e\right\} & = & 1 / 30 \\ (14) \quad\{b * \tilde{A}(\tilde{A} e * \tilde{A} e) * \tilde{A} e\} & = & 1 / 15 \\ (15) \quad\left\{b * \tilde{A}^{2} e * \tilde{A} e\right\} & = & 1 / 20 \\ (16) \quad\left\{b * \tilde{A}^{2} * \tilde{A} e * \tilde{A} e\right\} & = & 1 / 10 \\ (17) \quad\{b * \tilde{A} e * \tilde{A} e * \tilde{A} e * \tilde{A} e\} & = & 1 / 15\end{array}$

which

$$
\tilde{A}=\left(\begin{array}{cccccc}
0 & 0 & 0 & 0 & 0 & 0 \\
a_{21} & 0 & 0 & 0 & 0 & 0 \\
a_{31} & a_{32} & 0 & 0 & 0 & 0 \\
a_{41} & a_{42} & a_{43} & 0 & 0 & 0 \\
a_{51} & a_{52} & a_{53} & a_{54} & 0 & 0 \\
a_{61} & a_{62} & a_{63} & a_{64} & a_{65} & 0
\end{array}\right) \text { and } e=(1, \cdots, 1)^{t}
$$

The analytical solution of this system of equations is an extraordinary difficult issue and in the general cas this system of equation is not solved yet but only the varieties of solutions are particulary known (Butcher,1964). These particular solutions exist allow by the use of "simplifying assumptions" which allow to reduce the system of equation. This family of solution is parametrically represent according to the free variable which are $c_{2} ; c_{3} ; c_{4} ; c_{5}$ and $a_{43}$. Two main characteristics 
distinguish these solutions. These are the following cases:

$$
\begin{array}{r}
\text { First case }: b_{2}=0 ; \\
\text { Second case }: b_{2} \neq 0 .
\end{array}
$$

The property of these solutions has been isolated to the only case $b_{2}=0$ in this paper. The analytical expressions of solutions which result from the system are quite cumbersome and cannot be explicitly given in this work; the algorithm MAPLE can systematically expressed the values of these variables by free variables.

A few later, in the work in (Cassity,1969),we have found a family of the most general solutions. The expressions which result are more complex and contain the irrational of quadratic irrationality step.

Recently, there had obvious analytical expressions obtained via D. Werner's Maple.For others results, see for instance (Khashin,2013).

If we want to obtain a method of order 6 with 7 stages, then you must add 20 other equations to the previous system. It is clear enough that such a system is insoluble that is to say the additional equations do not take simultaneously 0 as the value. But we can try to minimize the members of left of these equations by using the value of $S^{2} . S^{2}$ is used to evaluate the quality of the method of Runge-Kutta .

The methods of RK of order 5 available in (Khashin,2013), have been achieved with aid of MAPLE functions:

$$
\begin{aligned}
\operatorname{RK} 56\left(c_{2}, c_{3}, c_{4}, c_{5}, b_{2}, a_{43}\right) & \text { with } b_{2}=0 \\
\operatorname{RK56} a\left(c_{2}, c_{3}, c_{3}, c_{4}, c_{5}, b_{2}, a_{43}\right) & \text { with } b_{2} \neq 0
\end{aligned}
$$

In the first case, all the coefficients of the matrix are rational numbers but the second case gives very large family of the solutions.The coefficients of the matrix are quadratic irrational numbers depending on a parameter and can be undetermined if the expression which is found under the radical is negatif. When $b_{2}=0$, we obtain exactly the coefficients of first case.

The formula obtained by the method of RK56a are too complex for the analytic study of the expression of $S^{2}$. That is why, we first study the method of RK56. In this case it is possible to obtained the explicit obvious expression and rational of $S^{2}$ by the parameters $c_{2}, c_{3}, c_{4}, c_{5}, a_{43}$. The formula is too wide to be written here, because the numerator is degree 10 and contains 144 monomials.however the system of computer algebra can be said that it works efficiently.

\section{Minimization}

In the denominator of the function error constructed there is an expression of the shape $c_{4}^{2}\left(c_{3}-c_{4}\right)^{2}$. It permits to show that $c_{4} \neq 0$ and $c_{3} \neq c_{4}$. However the methods of RK 56 are challenge deny to the following restrictions only:

$$
c_{i} \neq 0, \quad c_{i} \neq 1, \quad c_{3} \neq c_{4}, \quad c_{3} \neq c_{5}, \quad c_{4} \neq c_{5}
$$

Besides in the calculations, all the values of the variables $c_{i}$ must be comprised between the interval $[0 ; 1]$.

Dependence of $a_{43}$ : we notice that the numerator of the rational function which is being analysed depends on the square of $a_{43}$ and the main coefficient is always non negative. Since the denominator shape $c_{4}^{2}\left(c_{3}-c_{4}\right)^{2}$ doesn't depend on $a_{43}$ at all, the minimum is unique and is reached to $a_{43}$, that is a rational function of other variable. After replacing it in $S^{2}$, we get a new rational function again $S_{1}^{2}\left(c_{2}, c_{3}, c_{4}, c_{5}\right)$ that doesn't depend anymore on $a_{43}$.

Dependence of $c_{4}$ : we notice that the denominator of this rational function doesn't depend on $c_{4}$, and the numerator depends on the square of $c_{4}$ and once again the first coefficient is always greater than zero. Therefore the minimal value is obtained in $c_{4}$ that can be easily found. By replacing it in $S_{1}^{2}$, we get once again rational function $S_{1}^{2}\left(c_{2}, c_{3}, c_{4}\right)$ that depends more on $c_{4}$. The constructed rational function is not too complex. Its denominator is of degree 10 and is composed of 75 members. the numerator is of degree 11 and is composed 159 members.

The problem amounts to consists in finding the minimum of a rational function of three variables in the unit cube. The value of the $S_{2}^{2}$ function maybe arbitrarily small, but some coefficients of the methods will increase indefinitely in absolute value. Therefore, the problem will be complicated, we require that all coefficients of the method don't gobeyond a certain constant of 20 for example.

Optimum 1: The direct checking shows that the values close to the optimum will be the following :

$$
c_{2}=2 / 23, \quad c_{3}=12 / 37, \quad c_{4}=27 / 29, \quad c_{5}=199 / 200, \quad a_{43}=24 / 5 .
$$

Here is the resulting Butcher table : 


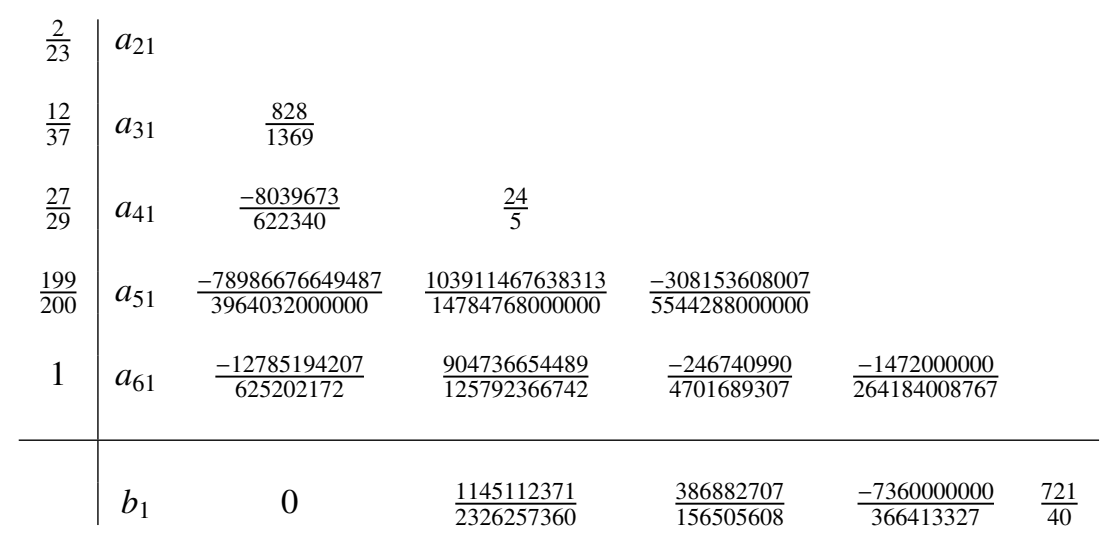

However $a_{k 1}=c_{k}=\sum_{i=2}^{k-1} a_{k i}$ and $b_{1}=1-b_{3}-b_{4}-b_{5}-b_{6}$

Therefore with six numbers after the comma, the table of Butcher becomes:

\begin{tabular}{l|cccccc}
0.869565 & 0.869565 & & & & & \\
0.324324 & -0.280496 & 0.604821 & & & & \\
0.931034 & 9.049492 & -12.918400 & 4.800000 & & & \\
0.995000 & 13.948144 & -19.925800 & 7.028270 & -0.055580 & & \\
1.000000 & 14.315445 & -20.449700 & 7.192300 & -0.052479 & -0.005572 & \\
\hline & & 0 & 0.492260 & 2.472000 & -20.086610 & 18.025000
\end{tabular}

So $S^{2} \approx 2.18 \times 10^{-9}$.

Optimum 2: The maximal value of the absolute value of the coefficients of matrix $A$ is greater to 20 . It will lead to an increase of the error of rounded. So we present another method with a slightly bigger value of the $S$ function but more smaller in absolute value. The coefficients used are :

$$
c_{2}=1 / 6, \quad c_{3}=12 / 37, \quad c_{4}=15 / 16, \quad c_{5}=74 / 75, \quad a_{43}=11 / 24
$$

So $S^{2}=9.54 \times 10^{-9}$ and the table of Butcher will be in this case :

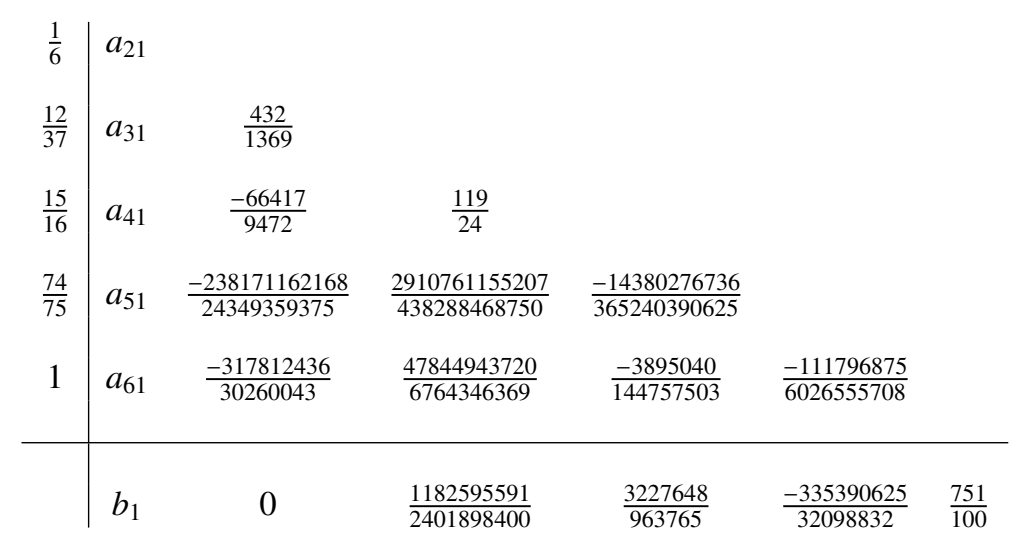

Therefore with six numbers after the comma, the table of Butcher becomes : 


\begin{tabular}{c|ccccccc}
0.166667 & 0.166667 & & & & & & \\
0.324324 & 0.008765 & 0.315560 & & & & \\
0.937500 & 2.99110 & -7.0119 & 4.95833 & & & \\
0.986667 & 4.16625 & -97814 & 6.64120 & -0.03937 & & \\
1.000000 & 4.47506 & -10.5027 & 7.07311 & -0.02691 & -0.0185507 & \\
\hline & 0.09733 & 0 & 0.49236 & 3.34900 & -10.4487 & 7.51
\end{tabular}

In comparison with the method of Dormane-Prince which is very after used (Wanner,Hairer,Nersett,2000) with

$$
c_{2}=1 / 5, c_{3}=3 / 10, c_{4}=4 / 5, c_{5}=8 / 9, a_{43}=32 / 9
$$

the value of $S^{2}$ is $0.25 \times 10^{-6}$, that is to say 90 times bigger.

For the method described in the book (Butcher,2008) for:

$$
c_{2}=1 / 4, c_{3}=1 / 4, c_{4}=1 / 2, c_{5}=3 / 4, a_{43}=1 / 2
$$

the value of $S^{2}$ is $4.9 \times 10^{-6}$, that is 1750 times bigger.

For the method of Fehlbert (Wanner,Hairer,Nersett,2000) with

$$
c_{2}=1 / 4, c_{3}=3 / 8, c_{4}=12 / 13, c_{5}=3 / 4, a_{43}=0
$$

the value of $S^{2}$ is $24 \times 10^{-6}$, that is 8500 times bigger.

Let's remind that $S^{2}$ is equal to the total of the squares of the gaps which is the absolute value of the error which report is a little smaller: for Dormane-prince's method the error is about 10 times bigger; for the method described in the book of Butcher, it is 40 times and for the method of Fehlbert it is 90 times.

Dependence of $b_{2}$ : the formula of the $S^{2}$ function in the case $b_{2} \neq 0$ is very cumbersone. For example, the matrix A obtained with the help of Maple gives values of order of $2.10^{6}$ and it doesn't permit us to built the $S^{2}$ function in general. That is why studing entirely and analytically its behavior is not possible yet. But its value is easy to find for any particular or valid whole of free variables.

The dependence of $b_{2}$, let's consider a value of $b_{2}$ relatively to the other variables proposed in the formula (2). Here, let's notice that for small values of $b_{2}$ is absolute value lower to 0 , the formula under the radical is negative. Therefore a solution exists in the neighborhood of 0 when $b_{2} \geq 0$. The table below, present the relation between $10^{5} . S$ in relation with $b_{2}$ when

$$
c_{2}=2 / 23, \quad c_{3}=12 / 37, \quad c_{4}=27 / 229, \quad c_{5}=199 / 200, \quad a_{43}=24 / 5 .
$$

we study the negative and positive value of the square root separately :

\begin{tabular}{|c|c|c|}
\hline$b_{2}$ & $+\sqrt{D}$ & $-\sqrt{D}$ \\
\hline 0.000000 & 4.66828 & 4.66828 \\
0.000002 & 4.67505 & 4.66376 \\
0.000004 & 4.67852 & 4.66256 \\
0.000006 & 4.68146 & 4.66191 \\
0.000008 & 4.68413 & 4.66156 \\
0.000010 & 4.68464 & 4.66139 \\
0.000012 & 4.68902 & 4.66136 \\
0.000014 & 4.69132 & 4.66144 \\
0.000016 & 4.69356 & 4.66160 \\
0.000018 & 4.69574 & 1.66184 \\
0.000020 & 4.69788 & 4.66214 \\
\hline
\end{tabular}


From the table above, we notice that although we can decrease the value of $S$ by an appropriated choice of $b_{2}$, this reduction is not sufficient and doesn't compensate the complexity of the algorithm. A similar situation exists for other initial values of the variable $c_{2} ; c_{3} ; c_{4} ; c_{5}$ and $a_{43}$. That is why, for the coming we will limit ourselves to the solutions in the case $b_{2}=0$.

And in general, we can suppose that the optimal methods are obtained when $b_{2}$ takes some values close to 0 and so we limit ourselves only to the methods for which $b_{2}=0$. But to confirm this hypothesis, we need more important arguments.

\section{Field of Stability}

The order is not the only incoming important condition in game in the choice of a method of Runge-Kutta. The second important condition is the field of stability of the method.

Both methods are steady enough. Their area of stability is more sread than the method extensively used by Dormaneprince. See graph in figure 1.

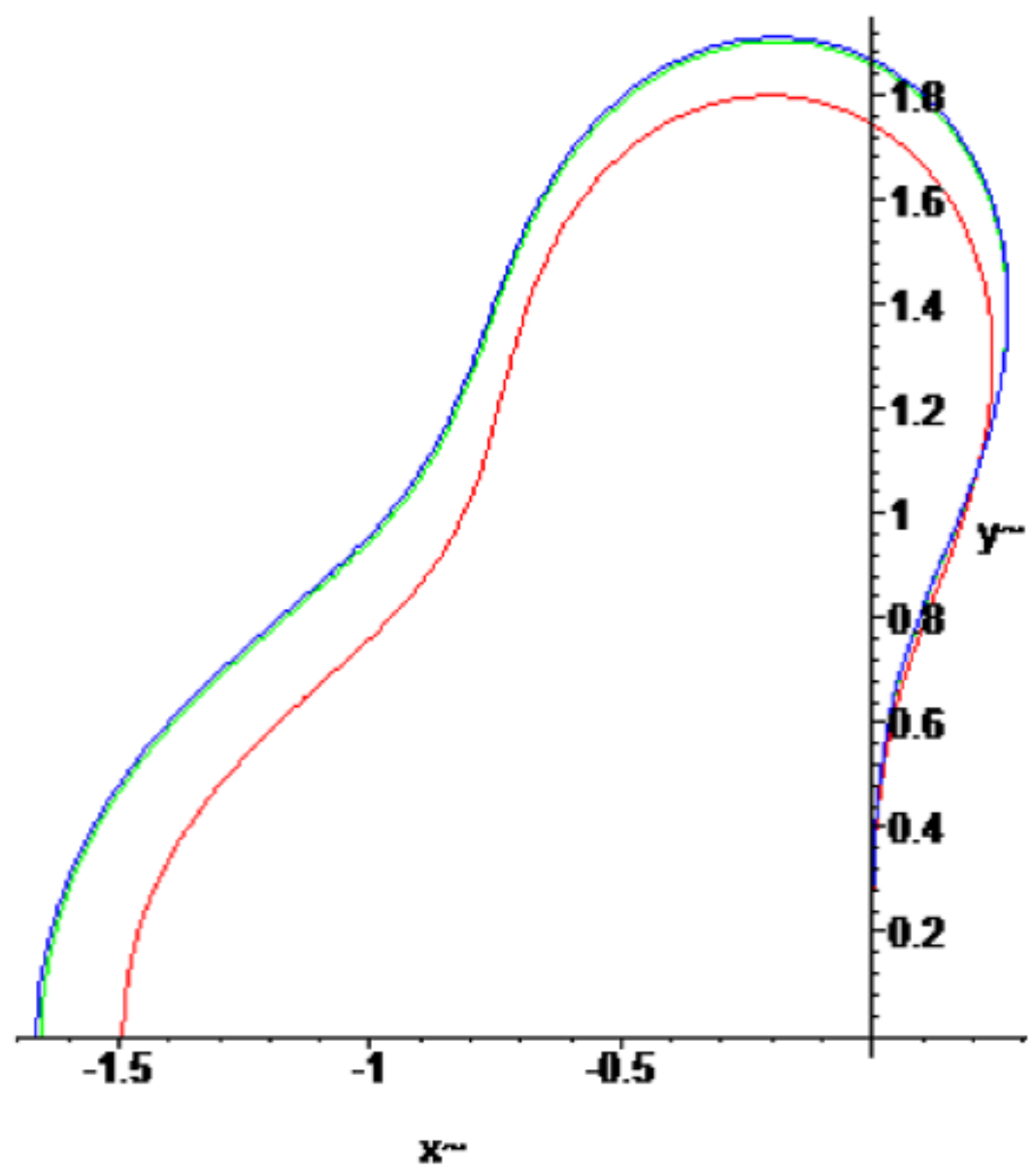

Figure 1. Graph 


\section{Evaluation of the Error With 3 Steps}

While solving the differential equations by a method of Runge-Kutta, it is very desirable to be able to estimate the local error in every stage. It allows us to select the size of suitable step automatically and to change it during the calculations. In the absence of such step of evaluation, we must choose it among a big margin, we mean a lot less that what is really necessary, it increases considerably the quantity of calculation. Usually, the evaluation of the truncation error is made by makes using" pair overlapped of RK methods, (Runge-Kutta pairs) they are less used.

In the work (Khashin,2014), we suggest a method to assess the local error by the classic methods of RK from the fourth order on the basis of three consecutive steps. The technique adopted can be applied to the methods of the fifth order.

We notice that for the methods of order 4 , we cannot get the evaluation of the error by using two steps, that is why we will search the evaluation of the error in three steps.

The table of Butcher of the method with 6 stages seems :

$$
\begin{array}{c|cccccc}
c_{2} & a_{21} & & & & & \\
c_{3} & a_{31} & a_{32} & & & & \\
c_{4} & a_{41} & a_{42} & a_{43} & & & \\
c_{5} & a_{51} & a_{52} & a_{53} & a_{54} & & \\
c_{6} & a_{61} & a_{62} & a_{63} & a_{64} & a_{65} & \\
\hline 1 & b_{1} & b_{2} & b_{3} & b_{4} & b_{5} & b_{6}
\end{array}
$$

We can consider the three steps of lengths fixed by this method with a step which can be considered like a method of 13 steps whose table of Butcher is the following:

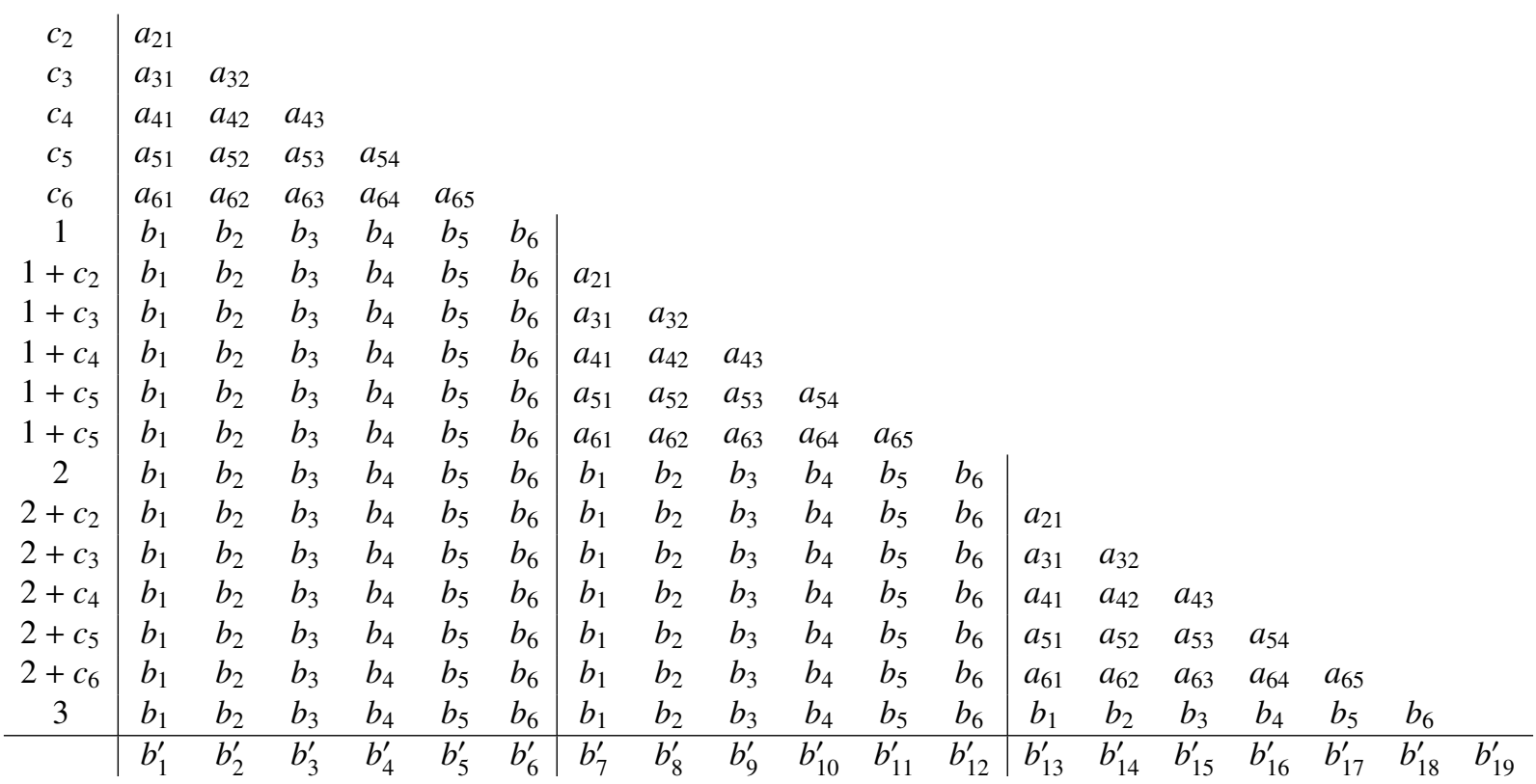

In the process of three consecutive steps of the original method of the RK, we get 19 intermediate vectors.

$$
\begin{array}{llllll}
k_{01} & k_{02} & k_{03} & k_{04} & k_{05} & k_{06} \\
k_{11} & k_{12} & k_{13} & k_{14} & k_{15} & k_{16} \\
k_{21} & k_{22} & k_{23} & k_{24} & k_{25} & k_{26} \\
k_{31} & & & & &
\end{array} .
$$

Their linear combination with the coefficients $b_{i}^{\prime}$ can give an estimate of the error with an accuracy of $O\left(h^{7}\right)$. As suggested in the work (Butcher,2000), we will search for $b_{i}^{\prime}$ in the form $b_{i}^{\prime}=b_{i}+$ beta $_{i}$. Thus, the formula for estimating the error vector $t o l$ will be

$$
t o l \approx h\left(\beta_{1} k_{01}+\cdots+\beta_{19} k_{31}\right) .
$$


The coefficients of $\beta_{i}$ are obtained as the solutions of a system of 37 equations of Butcher.The solution is unidimensional and $\beta_{19}$ can be chosen as a free variable. The choice of $\beta_{19}=0$ is particularly convenient because, the error can be found immediately after the 3 step, without calculating $k_{19}$.

For the method (optimum 1) we get the following coefficients:

$$
\begin{array}{ll}
\beta_{1}=890113 / 7737120, & \beta_{10}=-19357476133 / 1565056080, \\
\beta_{2}=0, & \beta_{11}=40215040000 / 366413327, \\
\beta_{3}=-8387174393 / 23262573600, & \beta_{12}=-7931 / 80, \\
\beta_{4}=5616469643 / 1565056080, & \beta_{13}=4695337 / 1934280, \\
\beta_{5}=-12379520000 / 366413327, & \beta_{14}=0, \\
\beta_{6}=12257 / 400, & \beta_{15}=-4673296433 / 5815643400, \\
\beta_{7}=1280389 / 7737120, & \beta_{16}=26681566 / 19563201, \\
\beta_{8}=0, & \beta_{17}=-5755520000 / 366413327, \\
\beta_{9}=-1454602201 / 4652514720, & \beta_{18}=721 / 50 .
\end{array}
$$

The maximum modulus coefficient is $\beta_{11} \approx 110$.

For the method (optimum 2) - such:

$$
\begin{array}{ll}
\beta_{1}=183937 / 1598400, & \beta_{10}=-81498112 / 4818825, \\
\beta_{2}=0, & \beta_{11}=3624901875 / 64197664, \\
\beta_{3}=-8661713653 / 24018984000, & \beta_{12}=-8261 / 200, \\
\beta_{4}=403456 / 81675, & \beta_{13}=970021 / 399600, \\
\beta_{5}=-18781875 / 1088096, & \beta_{14}=0, \\
\beta_{6}=12767 / 1000, & \beta_{15}=-4826268493 / 6004746000, \\
\beta_{7}=264469 / 1598400, & \beta_{16}=9279488 / 4818825, \\
\beta_{8}=0, & \beta_{17}=-126106875 / 16049416, \\
\beta_{9}=-1502216021 / 4803796800, & \beta_{18}=751 / 125,
\end{array}
$$

The maximum modulus coefficient is $\beta_{11} \approx 56$.

To complete, we give the same coefficients for the Dormane-Prince method (Hairer,Nersett and Wanner,1990)

$$
\begin{array}{ll}
\beta_{1}=261323 / 2602920, & \beta_{10}=-11183389 / 8329344, \\
\beta_{2}=0, & \beta_{11}=1648414071 / 1471517440, \\
\beta_{3}=-5768864 / 24142083, & \beta_{12}=-13896839 / 18220440, \\
\beta_{4}=-212473 / 520584, & \beta_{13}=109371217 / 83293440, \\
\beta_{5}=-588303 / 22992460, & \beta_{14}=0, \\
\beta_{6}=374033 / 2277555, & \beta_{15}=-2340314 / 24142083, \\
\beta_{7}=109371217 / 83293440, & \beta_{16}=-1685293 / 8329344, \\
\beta_{8}=0, & \beta_{17}=-187616169 / 1471517440, \\
\beta_{9}=-24427322 / 24142083, & \beta_{18}=749309 / 3644088,
\end{array}
$$

the maximum modulus coefficient is $\beta_{6} \approx 1.34$.

For the Butcher Method (Butcher,1964)

$$
\begin{array}{ll}
\beta_{1}=137 / 900, & \beta_{10}=-19 / 75, \\
\beta_{2}=0, & \beta_{11}=-296 / 225, \\
\beta_{3}=-16 / 45, & \beta_{12}=-77 / 180, \\
\beta_{4}=-1 / 75, & \beta_{13}=1117 / 450, \\
\beta_{5}=64 / 225, & \beta_{14}=0, \\
\beta_{6}=119 / 900, & \beta_{15}=-152 / 225, \\
\beta_{7}=119 / 900, & \beta_{16}=-2 / 15, \\
\beta_{8}=0, & \beta_{17}=-8 / 225, \\
\beta_{9}=-8 / 225, & \beta_{18}=14 / 225,
\end{array}
$$

The maximum modulus coefficient is $\beta_{13} \approx 2.5$. 
For the Felberg method, (Hairer,Nersett and Wanner,1990)

$$
\begin{array}{ll}
\beta_{1}=827 / 10800, & \beta_{10}=-1412671 / 615600, \\
\beta_{2}=0, & \beta_{11}=-296 / 2025, \\
\beta_{3}=-704 / 2375, & \beta_{12}=22 / 25, \\
\beta_{4}=81289 / 123120, & \beta_{13}=6401 / 2700, \\
\beta_{5}=64 / 2025, & \beta_{14}=0, \\
\beta_{6}=-34 / 125, & \beta_{15}=-1856 / 2375, \\
\beta_{7}=2159 / 10800, & \beta_{16}=37349 / 153900, \\
\beta_{8}=0, & \beta_{17}=-8 / 2025, \\
\beta_{9}=-256 / 475, & \beta_{18}=-16 / 125,
\end{array}
$$

The maximum modulus coefficient is $\beta_{10} \approx-2.3$.

\section{Conclusion}

So the smallest error of the indicated methods is the one of Dormane-prince and felhberg. Of course an example is not sufficient to appreciate fully the quality of the method. However, we got a part of their comparative description as shown by other experiences, the relation between the different methods of gotten precision is more or less the same for a lot of other methods.

\section{References}

Butcher, J. C. (1964). On Runge-Kutta processes of high order. J. Austral. Math. Soc., 4, 179-194. https://doi.org/10.1017/S1446788700023387

Butcher, J. C. (2008). Numerical methods for ordinary differential equations (2nd ed.). John Wiley \& Sons. https://doi.org/10.1002/9780470753767

Cassity, C. R. (1969). The complete solution of the fifth order Runge-Kutta equations. SIAM J. Numer. Anal., 6, 432-436. https://doi.org/10.1137/0706038

Hairer, E., Nersett, E., \& Wanner, G. (1990). Résolution des équations différentielles ordinaires. Les problèmes non rigides. Monde.

Jackiewicz, Z., \& Verner, J. H. (2000). Derivation and implementation of two-step Runge-Kutta pairs. Japan J. Indus. Appl. Math., 19, 227-248. https://doi.org/10.1007/BF03167454

Jackiewicz, Z. (2009). General Linear Methods for Ordinary Differential Equations. John Wiley \& Sons, Inc. https://doi.org/10.1002/9780470522165

Jérémy, C. (2011, Février). Méthodes numériques de ROCK4 pour la résolution d'équations différentielles et étude de l'algèbre de pré-Lie sous-jacente.

Khashin, S. I. (2014). Estimation de l'erreur de la méthode de Runge-Kutta classique. Journal of computational Mathematics and Physics Mathematics, 54(5), 24-32.

Khashin, S. I. (2013). List of some known Runge-kutta methods family. Retrieved from http://math.ivanovo.ac.ru/dalgebra/khashin/rk/sh_rk.html

Verner, J. H. (2010). Numerically optimal Runge-Kutta pairs with interpolants. Numerical Algorithms, 53, 383-396. https://doi.org/10.1007/s11075-009-9290-3

Wanner, G., Hairer, E., \& Nersett, S. P. (2000). Solving ordinary differential equations I. Nonstiff Problems. 2Ed. Springer-Verlag.

\section{Copyrights}

Copyright for this article is retained by the author(s), with first publication rights granted to the journal.

This is an open-access article distributed under the terms and conditions of the Creative Commons Attribution license (http://creativecommons.org/licenses/by/4.0/). 